\title{
The Future of the Physician Scientist \\ Presidential Address Delivered before the 76th Annual Meeting of the American Society for Clinical Investigation, Washington, DC, 4 May 1985
}

Robert M. Glickman

Department of Medicine, College of Physicians \& Surgeons of Columbia University, New York 10032

Science and medicine face so many difficult problems today that the choice is great when selecting a subject for this address. As academic physicians, we seem continually engaged in a struggle to secure the necessary resources to preserve our research and training missions. In addition, another set of threats is rapidly emerging, the economics of health care. Unlike any time in the recent past, events seem to be moving so fast now. The practice of medicine and the training of physicians as we have known them are in the process of substantial change. A representative sampling of one day's headlines in the press illustrates the many areas of concern which are entering the academic life of clinical departments throughout the country (Fig. 1). Topics for discussion at Grand Rounds are as likely to include an aspect of health care economics as an analysis of the genetic material of an Egyptian mummy. Patient-derived revenues, which are essential to the viability of most clinical departments, are seriously threatened. Thus the academic physician is charged by his department, his medical school, and his hospital with additional concerns relating to the economics of patient care. We are besieged from all sides by enormous forces.

During the 77-year history of our Society, external threats, as serious as they have been and continue to be, have eventually been resolved. Periods of disequilibrium, while damaging, are finite. Our history has been one of a continuing pursuit of biomedical research and there is no reason to believe that this enterprise will not continue in a vigorous manner-although, at any given period, growth may be restrained and anxiety may be appropriately high. We are currently in such a period. However, we have always been able to respond to external forces. We have always believed that we are engaged in essential work, research into the mechanisms of human disease and its eventual cure through understanding. We have always believed that an appropriate route to this goal has been the physician scientist. We have always believed, as an article of faith, that we, as physicians, have a special understanding, and more importantly, a mandate to investigate human illness. This confidence has enabled us to have a unity of purpose and to respond effectively to a variety of external threats.

It is therefore disturbing when these fundamental assumptions are questioned. Gordon Gill (1), a distinguished Professor of Medicine and a leader in biochemical research involving the cyclic nucleotides, raises serious questions concerning the viability of the physician as a scientist in a recent article entitled "The End of the Physician-Scientist?". He acknowledges the past achievements of American biomedical science but then notes:

In the seventeen years since I first attended a meeting in Atlantic City, the academic physicians had also been displaced from their dominant position in biomedical research, although most of them

J. Clin. Invest.

(c) The American Society for Clinical Investigation, Inc.

0021-9738/85/10/1293/04 \$1.00

Volume 76, October 1985, 1293-1296 did not yet recognize or acknowledge it. . . . Few people, if any, recognized that clinical investigation had become the development stage of science and that fundamental research and biological observations rarely originated any longer in medical schools. . . . but few seemed ready to acknowledge that the clinician scientist was now the clinician-applier of basic science or that the physician-basic researcher was unlikely to keep up, much less surpass, his colleagues in pure basic science.

He then identifies the factors responsible as mainly economic, with medical practice attracting more physicians, the decline of the National Institutes of Health as a post residency military service alternative, and the payback provision on training grants. More importantly, Gill discusses another factor:

The second force for change in the character of academic departments of medicine was perhaps more profound. The face and nature of molecular biology and true basic science changed with the advent of recombinant DNA technology; it changed so fast that, without a large cadre of bright young MD's in fellowship training, the revolution bypassed the medical world. . . . The advances in science will still be taught, but now those in academic medicine will look to their colleagues, not for exciting scientific ideas, but instead for ideas about delivery of health care, new diagnostic tests, new drugs for clinical use, faculty salary plans, hospitals, and medicare. As the private practice of medicine becomes more restrictive, academic medicine may again look more attractive to residents, but they will enter as academic physicians, not as physician-scientists.

A rather dismal diagnosis of what sounds like an incurable disease. It is tempting to dismiss these observations by seeking refuge in the mission of medicine and of medical research. This would be incorrect. Although I strongly disagree with the conclusions, they are rendered from a deep sense of concern by one of us, one of our most distinguished academic physicians, and raise substantive issues which deserve exploration.

I would therefore like to address the following points. First, what is clinical investigation? Has its fundamental nature changed, or as an important corrolary, will the Journal of Clinical Investigation need to be renamed? Second, is the physician essential in the process? Third, are clinical departments configured to encourage and support outstanding biomedical science?

Alfred E. Cohn wrote perhaps one of the best descriptions of clinical research as the dedication to Volume 1 of the Journal of Clinical Investigation entitled "Purposes in Medical Research" (2).

We have, as all those interested in the progress of medicine know, for some time been inquiring whether medicine is entitled to be called a science. To us the answer to this question is clear and unequivocal. It is clear because of the nature of the case. The phenomena of interest in medicine are the phenomena of disease as these are manifest in affected persons. They are phenomena which exist as concrete entities in nature, they are indivisible and they fall within the province of no other inquiry. They constitute the proper concern of medicine.

Thus Cohn defines the essential nature of clinical research as the phenomena of disease and its understanding. As such it is not encompassed as a primary objective of any individual 


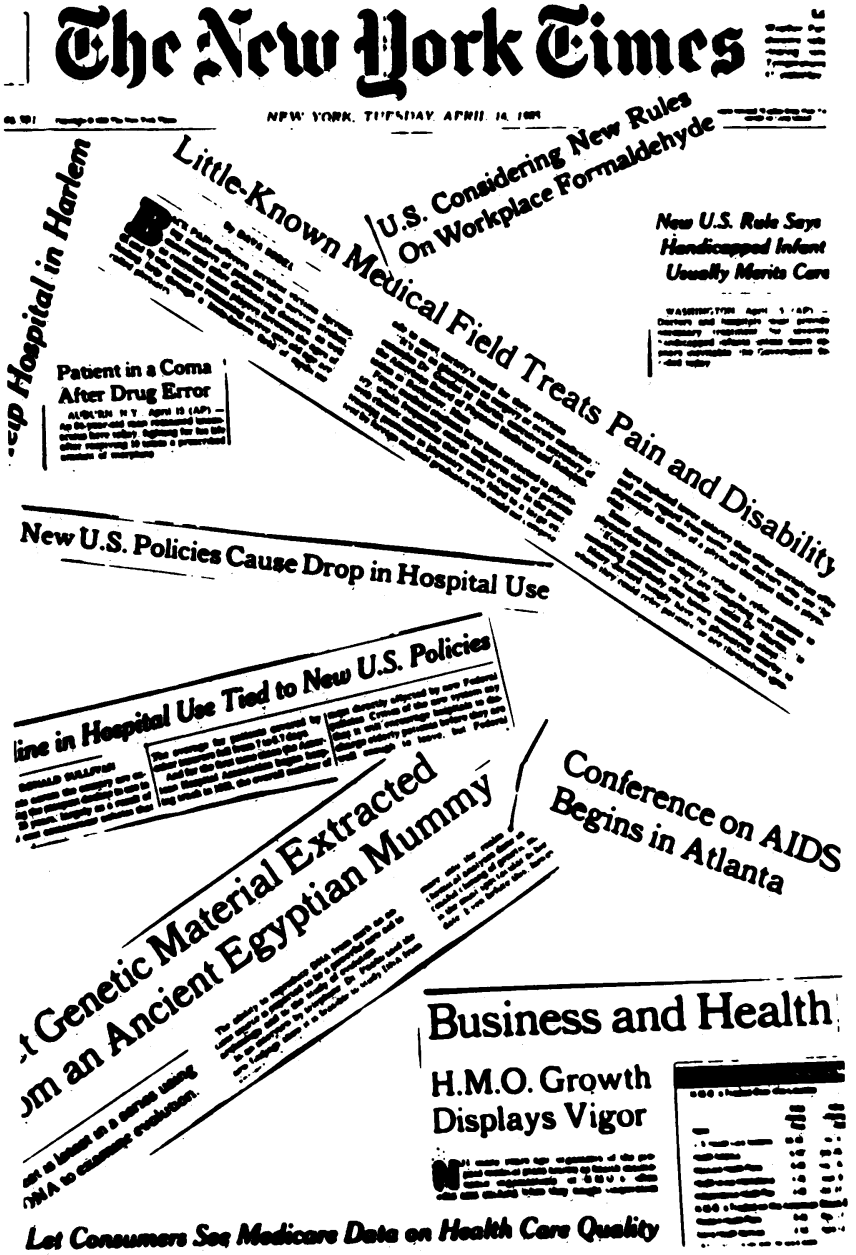

Figure 1. Contemporary issues in medicine as reported in the press.

scientific discipline. He also stresses that the flexibility to pursue investigation into the mechanisms of disease by whatever means are most appropriate is equally important. Thus, by definition, clinical investigation is applied. It is the application of state of the art technologic advances to human disease. I would contend that this has always been the case and that many of the most respected figures in academic medicine were wise enough to apply the latest advances in basic science to their particular problem. Did the elucidation of the pathway of cholesterol biosynthesis make the subsequent study of the regulation of cellular cholesterol homeostasis applied research? Did the discovery of prostaglandins and their biosynthesis make the exploration of their varied roles in health and disease into derivative work? Did the discovery of radioimmunoassay or the methodology of monoclonal antibody production make the application of these powerful technologies uninteresting in the investigation of numerous diseases? Of course not. Then why does it seem that the physician, who was formerly able to survey the best of technologies and productively apply them to his research, has been bypassed at this point in time as Gordon Gill suggests? Are the techniques and understanding of recombinant DNA and molecular biology too fundamental for the physician scientist to master and apply? Why have recent exciting advances in understanding of human disease been made as often in basic science departments as in clinical departments?

I believe that more than any other time in modern biomedical science we have encountered a fundamental, complex discipline which will not yield easily to a short intensive course in methodology. Our system of research training, which was basically organized through subspecialty fellowships in clinical departments, was unable to rapidly grasp the power of modern molecular biology and broadly apply it to the understanding of human disease. There were obvious notable exceptions where outstanding physician scientists with well-formulated research programs adapted molecular biology to probe the structure of abnormal hemoglobin genes, the insulin receptor, and the low density lipoprotein receptor. In general, however, most biomedical scientists were not so well-positioned and felt disenfranchised. Basic science departments could only offer limited collaborative efforts determined by the practicalities of interest and limited resources. Thus, perhaps for the first time, the physician scientist was unable to broadly apply the latest advances to his research. Rapidly coming to the realization that alternative approaches were necessary, physicians, both young and established, undertook the arduous task of training. This period of training was relatively unobtrusive and served to enforce the perception that the exciting science had left meetings of physician scientists and gone to Cold Spring Harbor. We all have observed that the physician scientist trained in modern biology is emerging; he often presents at plenary sessions such as ours with slides that defy all the suggested rules of composition. The effect was somewhat predictable and as noted by Samuel Meltzer (3), the founder of the American Society for Clinical Investigation:

Some older members complain that the papers presented at the meetings are getting above their heads. While this may be a fact, it cannot be the basis of complaint-the science of medicine is progressing.

The new vocabulary is becoming familiar. Exons and introns are not new subatomic particles and an LTR is not a new video cassette recorder. The tools of molecular biology are now yielding valuable insights into the mechanisms of disease, as is evidenced by the presentations at this meeting. Thus, like every technology before it, molecular biology will become part of the repertoire of those physician scientists who can profitably apply this discipline to solving the problems of human disease.

Is the physician necessary in this process? Is the essence of clinical research, the essence of the patient, represented by a small fragment of his genetic material spirited away to a molecular biology or biochemistry department to be expressed in an appropriate vector? Is the main role of the physician to collect and shepherd these materials through the myriad of regulations of Institutional Review Boards? Will we become the procurers of human materials? I can't believe that the same genetic material and indomitable spirit that conceived the Doc in the Box, outmaneuvered DRG's, and financed office nuclear magnetic resonance spectrometers would be relegated to such a passive role. It is inconceivable to me that the gains to be made through the application of these new insights will not involve the physician scientist in their most exciting and most meaningful applications.

Again, to quote Meltzer (4):

Who should be the men to carry out research in this field and what should be their qualifications? First it is essential that they have had a bringing up within medicine; their senses must have been filled up with thinking, worrying and brooding over practical and theoretical problems of clinical medicine. Second, they must have a training fitting them to carry out investigation in conformity with the requirements existing in all pure sciences-they must also have carried 
on serious investigations in one or more of these pure sciences, so as to become familiar with careful scientific methods and imbued with a scientific spirit. They will thus acquire the habits and the tastes of the investigator, the scientist which may stick to them for life.

Thus I strongly believe that if properly trained, we are the only biomedical scientists specifically charged with investigating illness. Certainly questions of basic biology will clearly overlap with any given basic science. However, like it or not, the basic science of disease is our task. We cannot be certain that our broad range of interests will be faithfully served by industry, venture capitalists, or a relatively small number of basic scientists interested in human disease.

Four years ago, in his Presidential Address (5), Dr. William Paul carefully analyzed why, when biomedical science was on the threshold of major new discoveries, were fewer physicians pursuing careers in clinical investigation. One of the main factors that he identified was the diminishing opportunities for young physicians to undertake the rigorous training necessary for a serious scientific career. During the past four years there has been some progress in this regard. In my experience, as chairman of the Department of Medicine in a large urban academic medical center, and that of many chairmen that I have queried, there has been a modest but definite shift in the interest of young physicians towards a career in academic medicine. The reasons for this are multiple, including growing constraints on the practice of medicine and the often cited oversupply of physicians. Therefore, Bill Paul's admonitions to create a suitable research training environment are even more cogent. The data seem clear. Success in achieving research support is directly proportional to the number of years of research training. It is hard to imagine how someone with no prior research experience and with less than three years of research training can compete for funding. The newly created Physician Scientist Training Program, which joins other training programs, seems ideal in this regard. It is an attempt to recreate a pattern of clinical and research training which was remarkably successful in training many of the members of this Society. Two years of house staff training were followed by a period of two or three years at the National Institutes of Health immersed in a research environment with minimal clinical responsibilities. In some cases, this was followed by a third year of clinical training or more commonly, entry directly into a combined clinical and research fellowship program. These returning nascent physician scientists, better rested, five to ten pounds heavier, and several years older served as imposing role models for younger house staff. The Physician Scientist Program, conceived by Dr. James Wyngaarden, offers M.D.'s, after two or three years of clinical training, five years of research training. Three of these are carried out in a basic science laboratory with the final two years spent in establishing a research effort back in the sponsoring clinical department. In most cases clinical fellowship training can be dispersed throughout the latter portions of this program. In my own institution, this program has been highly sought after and laboratories in every basic science department are willing hosts for these trainees. Why not! The brightest medical residents who have mastered the art of transferring Veterans of the Spanish/American war from our emergency room in the middle of the night, who without the aid of pencil or paper can immediately calculate the impact of a complex scheduling change on their vacation or elective time, or who can extemporaneously come up with ten reasons not to wear a tie (one of the positive attractions of becoming a molecular biologist) have always had, and continue to have, the right stuff to perform remarkably well in a challenging research environment. Such programs should be expanded. I believe the demand is there and growing. Once again we will become talent developers and not merely talent scouts, identifying promising housestaff early in their careers and guiding and sponsoring their training.

I would now like to turn to the question of the clinical department as a suitable and stimulating environment for the physician scientist. From what I have already described, it is apparent that a more prolonged period of research training will be required to equip the physician scientist. We all believe that these individuals should be protected in the early stages of their development; yet, we all can cite multiple examples where this is not the case. These new faculty members are often gifted teachers and clinicians, are excellent role models, and are usually in great demand. While a limited clinical commitment is both necessary and desirable, missing pieces of salary support must be provided by other mechanisms. If sufficient funds are not available to protect these individuals, then fewer people should be trained, but they must be properly trained. A variety of supplementary funding sources will need to be identified to insure that young faculty in critical phases of research training, be it basic or more clinical, are allowed to develop. An innovative program has recently been undertaken by the American Gastroenterological Association and the pharmaceutic industry where matching funds provide flexible, supplementary funding support for young investigators. There is more than one way to cure an ulcer!

Does a limited commitment of time to clinical activities in part nullify the effectiveness of the physician scientist as a bridge between clinical and basic science? I believe not. Clinical medicine and research have become too demanding for a single individual to pursue both with the necessary aggressiveness required in today's competitive market. At the present time and increasingly in the future, clinical departments will accommodate a wide diversity of interests (Fig. 2). This will give them their vitality and their longevity. They should be home to the physician pursuing both basic and more clinical research and to the practice of medicine. The common thread which links all these physicians revolves around human disease. A notable exception is the chairman who is too busy going to meetings to enjoy any of this. Even if limited, it is the unique relationship to the patient which distinguishes the physician scientist.

I also believe that we must provide increased core facilities to broadly support the basic research activities of our faculty. Core facilities and advanced technology may not generally be available to younger investigators and are often fully committed to a given program project, Specialized Centers for Research program, or restricted to members of a center. The techniques
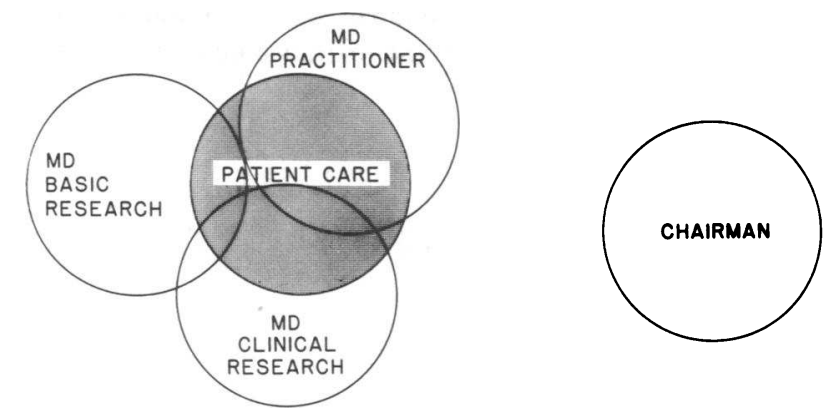

Figure 2. Relationships in a clinical department. 


\section{A Primer on Human DNA}

- $3,000,000,000$ units in a human cell (uncoiled $=2 \mathrm{~m}$ )

- 10,000,000 genes possible

- Information content comparable with a full set of Encyclopedia Britannica

- Only about $1 \%$ active (rest 'selfish'?)

- 100,000 proteins probably make up the constituents of the human body

- About 1,000 proteins have names and can be guessed to be present in the body

- About 100 proteins have been isolated and definitely characterized in humans

- About 10 human proteins have medical uses today

SCHEME I

of recombinant DNA are an excellent case in point. The lack of access to this expertise often results in unnecessary periods of "trial and error" for any investigator, young or established. Certain core functions, such as animal care facilities and clinical research centers, are properly provided by institutions since they provide common resources for investigators across departmental lines. Is the need any less persuasive for advanced methodological cores to support millions of dollars of funded research in many clinical departments? Serious consideration should be given to establishing funding mechanisms from the National Institutes of Health that are not tied to specific institutes, diseases, or programs but are viewed as a broad investment in research. Perhaps institutions with a certain amount of National Institutes of Health funding would be eligible and could propose cores of value to that particular university. Matching funds could be required from the institution, perhaps from indirect costs. This form of capital investment would be repaid many times over and is certainly required to preserve the research base of all universities. While efforts along these lines are being created by private sources, they are likely to be too few in number, involve mainly renowned scientists, and free access to them is uncertain.

Most importantly, born out of mutual needs, I see a growing collaborative relationship between clinical and basic science departments. As Joshua Lederberg pointed out (6), the task of exploring the expression of a possible 100,000 proteins by an enormous genome, selectively expressed, would require a $\$ 100$ billion dollar investment. (Scheme I). The magnitude of the enterprise demands a sense of priorities. I have addressed the scientific training of physicians but equally important is the exposure of basic scientists to important health problems. The realistic filter of human physiology and disease is necessary to focus this mission. There are growing numbers of basic scientists who have an increased interest in mechanisms of human disease and see the real benefits of being immersed in the intellectual environment of a clinical department while maintaining their ties to their basic department. Some rearrangement of traditional space assignments across divisional and departmental lines to reflect powerful research themes rather than administrative groupings is highly desirable in this regard and would do much to promote these interactions. The clinical department is a superb place for these interactions to take place.

Finally, I see the clinical and research missions of university hospitals and medical schools coming closer together than ever before. As stressed by Dr. Arnold Relman, our teaching hospitals facing financial constraints and growing competition in all sectors have been forced to identify those factors which make them unique and set this class of hospitals apart from other hospitals (7). Academic institutions will have difficulty competing on purely economic grounds with hospitals with less complex structures and simpler missions. In this climate, it is the research and teaching mission of the academic medical center which must be stressed and protected, as much for the self interest of the institution as for the societal benefits of research and education. Thus, the physician scientist will find his efforts increasingly appreciated and increasingly important in the fundamental thinking of the university hospital. This will be particularly true in a host of clinical research activities essential to maintain innovative patient care programs.

In conclusion, I have tried to define clinical research in the context of the physician scientist, explore some aspects of training and faculty development in a clinical department, and suggest how competitive forces will serve to encourage the development of the physician scientist. There is little question that events will become more complex as pressures from a variety of sources intensify. I have great faith, however, that we can successfully cope with these external forces as long as our confidence in ourselves is maintained. I would ask you all to return to your institutions and remove all "Do not resuscitate" orders from the physician scientist. The reports of his death have been greatly exaggerated; he is not dead, nor will we let him die.

\section{References}

1. Gill, G. N. 1984. The end of the physician-scientist? Am. Scholar 53:353-368.

2. Cohn, A. E. 1919. Purposes in medical research. J. Clin. Invest. $1: 1-11$.

3. Meltzer, S. J. 1915. The aims of the Association of American Physicians. Trans. Assoc. Am. Physicians (Phila.). 30:1-6.

4. Meltzer, S. J. 1909. The science of clinical medicine: What it ought to be and the men to uphold it. JAMA (J. Am. Med. Assoc.) 53:508512.

5. Paul, W. E. 1981. Clinical investigation-on the threshold of a golden era? J. Clin. Invest. 68:823-826.

6. Lederberg, J. 1982. Cycles in Biomedical Research in Academic Medicine, Present and Future. J. Z. Bowers and E. E. King, editors. Rockefeller Archive Center Conference.

7. Relman, A. S. 1984. Who will pay for medical education in our teaching hospitals? Science (Wash. DC). 226:20-23. 